\title{
BMJ Open Biomechanical factors associated with the development of tibiofemoral knee osteoarthritis: protocol for a systematic review and meta-analysis
}

\author{
Joyce A C van Tunen, ${ }^{1}$ Andrea Dell'Isola, ${ }^{2}$ Carsten Juhl, ${ }^{1,3}$ Joost Dekker, ${ }^{4}$ \\ Martijn Steultjens, ${ }^{2}$ Hans Lund ${ }^{1,5}$
}

To cite: van Tunen JAC, Dell'Isola A, Juhl C, et al. Biomechanical factors associated with the development of tibiofemoral knee osteoarthritis: protocol for a systematic review and meta-analysis. BMJ Open 2016:6:e011066.

doi:10.1136/bmjopen-2016011066

- Prepublication history and additional material is available. To view please visit the journal (http://dx.doi.org/ 10.1136/bmjopen-2016011066).

Received 6 January 2016 Revised 28 March 2016 Accepted 21 April 2016
CrossMark

For numbered affiliations see end of article.

Correspondence to Joyce AC van Tunen; jvtunen@health.sdu.dk

\begin{abstract}
Introduction: Altered biomechanics, increased joint loading and tissue damage, might be related in a vicious cycle within the development of knee osteoarthritis (KOA). We have defined biomechanical factors as joint-related factors that interact with the forces, moments and kinematics in and around a synovial joint. Although a number of studies and systematic reviews have been performed to assess the association of various factors with the development of KOA, a comprehensive overview focusing on biomechanical factors that are associated with the development of KOA is not available. The aim of this review is (1) to identify biomechanical factors that are associated with (the development of) KOA and (2) to identify the impact of other relevant risk factors on this association.
\end{abstract}

Methods and analysis: Cohort, cross-sectional and case-control studies investigating the association of a biomechanical factor with (the development of) KOA will be included. MEDLINE, EMBASE, CINAHL and SPORTDiscus will be searched from their inception until August 2015. 2 reviewers will independently screen articles obtained by the search for eligibility, extract data and score risk of bias. Quality of evidence will be evaluated. Meta-analysis using random effects model will be applied in each of the biomechanical factors, if possible.

Ethics and dissemination: This systematic review and meta-analysis does not require ethical approval. The results of this systematic review and meta-analysis will be disseminated through publications in peerreviewed journals and presentations at (inter)national conferences.

Trial registration number: CRD42015025092.

\section{INTRODUCTION}

The aetiology of osteoarthritis is known to be both biological and mechanical. ${ }^{1}$ Although the sequence is unknown, increased joint loading and altered biomechanics might lead to tissue damage. This might deteriorate

\section{Strengths and limitations of this study}

- To the best of our knowledge, performing this review will result in the first article giving an overview of the existing evidence of biomechanical factors that are associated with the development of knee osteoarthritis (KOA)

- This review does not assess the mechanism of how the biomechanical factors are related to the development of KOA.

- This is a protocol article. Results of the systematic review and meta-analysis will be published in a different article.

into structural changes, potentially with symptoms of osteoarthritis such as pain and activity limitations. In turn, structural changes might lead to further altered biomechanics, forming a vicious cycle.

A recent systematic review showed that several other risk factors, such as age, gender, body mass index (BMI) and previous knee injury, were associated with the development of knee pain or knee osteoarthritis (KOA), in those aged 50 years and over. ${ }^{2}$ In an editorial accompanying this review, Zhang $^{3}$ suggested that a systematic review and meta-analysis in biomechanical risk factors should also be performed. We have defined biomechanical factors as joint-related factors that interact with the forces, moments and kinematics in and around the knee joint. Current research into biomechanical factors focuses on four main biomechanical impairments relevant to tibiofemoral KOA, that is, skeletal malalignment, impaired proprioception, muscle dysfunction and laxity (table 1). Skeletal malalignment refers to abnormal alignment of or deformity within the knee joint. Valgus and varus malalignment might lead to increased loads in the lateral and medial compartment of the 
Table 1 Biomechanical impairments and risk factors relevant to the development of KOA, subdivision, and measurement

\begin{tabular}{|c|c|c|c|}
\hline & Impairment/risk factor & Subdivision & Measurement \\
\hline \multirow[t]{14}{*}{ Abnormal loading } & & & Moments, KRF, KCF, thrust \\
\hline & Skeletal malalignment & & Leg alignment (eg, HKA), Q-angle \\
\hline & \multirow[t]{2}{*}{ Muscular dysfunction } & Muscular & Strength, HQ-ratio \\
\hline & & Neurological & $\begin{array}{l}\text { Activation pattern, RFD, cocontraction, } \\
\text { coactivation }\end{array}$ \\
\hline & \multirow[t]{2}{*}{ Impaired proprioception } & Joint position sense & Error, accuracy \\
\hline & & Joint movement sense & Threshold \\
\hline & \multirow[t]{2}{*}{ Laxity } & Anterior-posterior & Joint line opening with stress \\
\hline & & Varus-valgus & Joint line opening with stress \\
\hline & \multirow[t]{2}{*}{ High BMI } & Categorical $^{\star}$ & Eg, obese, $\mathrm{BMl}>30$ \\
\hline & & Continuous & $\mathrm{BMI}$ \\
\hline & Gender & Categorical $^{*}$ & Male, female \\
\hline & Previous knee injury† & Categorical $^{*}$ & $\begin{array}{l}\text { Eg, ligament rupture, meniscal lesion, } \\
\text { tibia plateau fracture }\end{array}$ \\
\hline & \multirow[t]{2}{*}{ Age } & Categorical $^{*}$ & Eg, middle-aged, elderly \\
\hline & & Continuous & Age \\
\hline
\end{tabular}

${ }^{*}$ Comparable categorical risk factors will be grouped in analyses, based on decision by the two reviewers.

†Studies should not be influenced by rehabilitation or surgical treatment.

$\mathrm{BMI}$, body mass index; HKA, hip-knee-ankle-angle; H/Q ratio, Hamstrings/Quadriceps ratio; KCF, knee contact force; KOA, knee

osteoarthritis; KRF, knee reaction force; RFD, rate of force development.

tibiofemoral joint, respectively, and thus a possible increased risk for the development of KOA. Impaired proprioception refers to a deterioration of the senses of knee joint position and movement, or a primary neurological defect. This might lead to more excessive movements, decreased stabilisation during stance and decreased coordination of complex movement systems and precise knee joint motions. Deterioration of those three functions of knee proprioception might lead to increased joint loading and structural changes. ${ }^{4}$ Muscle dysfunction refers to a loss of muscle strength, or muscle weakness, to loss of muscle endurance, and to changed muscle activation patterns for the muscles that act on the knee joint. It is suggested that the knee extensors protect the joint during loading and movement, by absorbing shocks and stabilising the knee. ${ }^{5}$ On the contrary, higher strength could also lead to higher loads in the knee joint because of joint space narrowing induced by muscle strength. Laxity refers to a loss of passive joint stabilisation due to the inability of passive structures in and around the knee (knee ligaments, cruciate ligaments and capsule) to provide an adequate counterbalance to the mechanical forces acting on the knee during activity. For example, lateral laxity allows the lateral femoral condyle to 'lift of' the tibial plateau, which increases the medial joint loading. ${ }^{6}$ Laxity may adversely affect other biomechanical factors. ${ }^{7}$ Alteration in these four biomechanical impairments will impact on the loading of the knee. Abnormal loading is associated with imbalances that may eventually lead to KOA. ${ }^{8}$ Within the development of KOA, biomechanical factors and other risk factors will interact with each other. For example, gender is associated with malalignment, and previous knee injury has been shown to increase laxity. Although it is not possible to reduce the risk to develop KOA based on other risk factors, it might be possible to reduce the additional risk of the biomechanical factors by preventive strategies (eg, knee braces, insoles or physical therapy).

The relationship between biomechanical factors and the development of KOA has been indicated in several biomechanical studies and systematic reviews, but there is no systematic review available that contains an overview of all available evidence of the influence of particularly biomechanical factors on the development of KOA. ${ }^{5-11}$ Although each biomechancial factor will be assessed with several different methods, and most studies most probably have a cross-sectional study design, we expect to be able to give an overview of biomechancial factors that are associated with (the development of) KOA. Such an overview may be used to identify persons at high risk of developing KOA. Several strategies like physical therapy, knee braces or insoles might prevent the development of KOA in those persons by interacting on the biomechanical factors. In addition, an overview provides information that can be used to base the rationale behind strategies to prevent the development of KOA on and to identify the focus of future biomechanical and clinical studies.

\section{Objectives}

The aim of this review is (1) to identify biomechanical factors that are associated with (the development of) KOA and (2) to identify the impact of other relevant risk factors on this association. Therefore, the proposed systematic review will answer the following questions:

1. Which are the biomechanical factors that are associated with (the development of) KOA?

2. Is the association of these biomechanical factors with (the development of) KOA mediated by other risk factors for KOA (ie, high BMI, female gender, history of previous knee injury and higher age)? 


\section{METHODS AND ANALYSIS}

This review protocol is registered with the International Prospective Register of Systematic Reviews at the National Institute for Health Research and Center for Reviews and Dissemination (CRD) at the University of York (registration number: CRD42015025092). ${ }^{12}$ This systematic review is reported following the Preferred Reporting Items for Systematic Reviews and Meta-analyses Protocols guidance. ${ }^{13}$ The systematic review will be reported following the Preferred Reporting Items for Systematic Reviews and Meta-analyses (PRISMA) guidance. ${ }^{14}$

Studies will be selected according to the criteria outlined below.

\section{Type of studies}

Studies will be eligible if they are cohort, cross-sectional or case-control studies. The cohort studies can either be prospective or retrospective. All cohort studies should have a follow-up period of at least 1 year. Studies should not be influenced by rehabilitation or surgical treatment.

\section{Type of participants}

Included studies will be cohort studies examining participants who do not have KOA at baseline but who have KOA at the follow-up measurement, and participants who do not have KOA at both baseline and follow-up. Those two groups will be referred to as 'developing KOA' and 'not developing KOA'. Other included studies will be case-control and cross-sectional studies that include both participants with KOA and healthy controls. Participants have KOA if one of the criteria described in table 2, or an outcome measure related to the criteria, is fulfilled. Both idiopathic and secondary KOA are eligible. Participants can have osteoarthritis in one or both knees, although data should be analysed for only one knee, in order to examine independent associations with KOA. Studies examining participants with both osteoarthritis of the hip and the knee will only be included if separate data of participants with KOA is available. Studies examining participants already having KOA at baseline (cohort studies) or studies examining 'healthy' controls having KOA in either the index or the contralateral knee (cross-sectional or case-control studies) according to aforementioned criteria will be excluded. Studies examining participants with osteoarthritis in the patellofemoral joint only will be excluded. Data regarding patellofemoral osteoarthritis will not be used, and we will exclude data of individuals stated to have a combination of patellofemoral and tibiofemoral osteoarthritis. Cohort studies examining incidence of KOA in other populations (eg, rheumatoid arthritis and hypermobility) will be included, although only data regarding $\mathrm{KOA}$ will be used.

\section{Type of biomechanical factors}

Studies exploring the association of a biomechanical factor with (the development of) KOA will be reviewed. The biomechanical factors will be grouped into one
Table 2 Criteria of osteoarthritis, and hierarchy of definitions (ranking: A (high) - J (low))

\begin{tabular}{|c|c|c|}
\hline 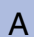 & Clinical KOA & AcCutuit \\
\hline & $\begin{array}{l}\text { Radiological } \\
\text { KOA }\end{array}$ & $\begin{array}{l}\text { Kellgren and Lawrence grade } \geq 2 \text {, or } \\
\text { grade } \geq 2 / \text { osteophytes, or Ahlback } \\
\text { grade } \geq 1\end{array}$ \\
\hline & $\begin{array}{l}\text { Radiological } \\
\text { KOA }\end{array}$ & $\begin{array}{l}\text { OARSI atlas criteria: sum of } \\
\text { osteophytes or JSN } \geq \text { grade } 2 \text {, or } \\
\text { grade } 1 \mathrm{JSN} \text { in combination with } \\
\text { grade } 1 \text { osteophyte }\end{array}$ \\
\hline & Clinical KOA & Knee pain and $\geq 50$ years \\
\hline & $\begin{array}{l}\text { Radiological } \\
\text { KOA }\end{array}$ & $\begin{array}{l}\text { Radiographic signs*; only if the } \\
\text { authors report the participants to } \\
\text { have KOA }\end{array}$ \\
\hline & $\begin{array}{l}\text { Surgery due to } \\
\text { KOAt }\end{array}$ & Arthroscopy used to describe KOA \\
\hline & $\begin{array}{l}\text { Surgery due to } \\
\text { KOA† }\end{array}$ & Osteotomy due to \\
\hline & $\begin{array}{l}\text { Surgery due to } \\
\text { KOA† }\end{array}$ & Total knee replacement due to KOA \\
\hline & $\begin{array}{l}\text { KOA defined by } \\
\text { MRI }\end{array}$ & $\begin{array}{l}\text { KOA signs on MRI; only if the } \\
\text { authors report the participants to } \\
\text { have KOA }\end{array}$ \\
\hline & Clinical KOA & $\begin{array}{l}\text { Participants report to have KOA } \\
\text { diagnosed by physician }\end{array}$ \\
\hline \multicolumn{3}{|c|}{$\begin{array}{l}{ }^{*} \text { Eg, osteophytes, cartilage damage, joint space narrowing, bone } \\
\text { marrow oedema. } \\
\text { tOnly as outcome measure of cohort study. } \\
\text { ACR, American College of Rheumatology; JSN, joint space } \\
\text { narrowing; KOA, knee osteoarthritis; OARSI, Osteoarthritis } \\
\text { Research Society International. }\end{array}$} \\
\hline
\end{tabular}

group referring to abnormal loading, and four main biomechanical impairments relevant to KOA (see table 1). Table 1 also describes frequently used ways to measure biomechanical factors. The possible inclusion of measurements of biomechanical factors not listed in table 1 will be discussed by the reviewers (JT and ADI). Only biomechanical factors directly related to the knee joint will be taken into account. Joint angles during activities will be excluded due to limited reliability of these measurements. Measurements of moments, knee reaction forces and knee contact forces will be included as measurements of abnormal loading, in contrast with measurements of ground reaction forces and centre of pressure, because only the first gives a comprehensive estimate of the loading of the knee. The influence of other risk factors on the association of biomechanical factors with (the development of) KOA will also be reviewed. Studies that only explore non-biomechanical risk factors will be excluded from this review.

\section{Types of outcome measure}

KOA in included studies can be defined by clinical, radiological, MRI or surgical criteria (see table 2).

\section{Publication year and language}

There will be no restriction on publication year and language. 


\section{Sources and search strategy}

The following databases will be searched from their inception until August 2015: MEDLINE via Pubmed, EMBASE via OVID, CINAHL (including preCINAHL) via EBSCO and SPORTDiscus via EBSCO. Reference lists from included studies and identified relevant reviews, textbooks and clinical guidelines will be searched for relevant references. A citation search will be performed on highly relevant studies. Experts in the field will be asked for relevant references to ensure literature saturation.

Literature search strategies will be developed using medical subject headings (MeSH) and text words related to osteoarthritis, biomechanical factors and study types. Search terms from other relevant reviews will be identified and will be complemented with additional terms for biomechanical factors. The MEDLINE search strategy is included in online supplementary appendix 1 , and will be adjusted in order to apply it to other databases.

\section{Study inclusion and exclusion criteria}

The inclusion criteria will be:

- The study assesses the association of a biomechanical factor with the development of KOA, and possibly the impact of other risk factors on this association.

- KOA is defined as tibiofemoral osteoarthritis, either idiopathic or secondary.

- A biomechanical factor is a knee joint-related factor that interacts with the forces, moments and kinematics in and around the knee joint.

- The study is a cohort study:

- Participants who do not have KOA at baseline but who have KOA at the follow-up measurement, and participants who do not have KOA at both baseline and follow-up.

or

- The study is a case-control study or a cross-sectional study:

- Participants with and without KOA.

The exclusion criteria will be:

- The study includes only participants with patellofemoral osteoarthritis.

- The study does not distinguish between hip osteoarthritis and $\mathrm{KOA}$.

- The study is influenced by rehabilitation or surgical treatment.

\section{Data management}

References from all searches will be uploaded into EndNote (X7) bibliographic software (Thompson Reuters, Philadelphia, Pennsylvania, USA). Duplicates will be removed with the Systematic Review Assistant-Deduplication Module. ${ }^{15}$ The remaining duplicates will be removed with EndNote duplicate removal, and manually by screening for duplicates, while the list of references will be sorted alphabetically based on author. All full text files will be stored in EndNote. For all studies reviewed in full text, a form regarding eligibility criteria check will be stored. Data from included studies will be entered into a data extraction form in Microsoft Excel. If a meta-analysis is eligible, the data will be transferred to STATA software (V13.0 or later, StataCorp LP) to do statistical analysis.

\section{Selection process}

Two members of the study team (JT and ADI) will independently screen titles and abstracts of the studies obtained by the search strategy. The full text of any study will be obtained if it was judged eligible by at least one of the reviewers. Then, the two reviewers will use a standardised form to select studies eligible for inclusion in the review. Consensus on inclusion will be reached by discussion. Reasons for excluding studies based on the full text will be recorded. When more than one study is based on the same population and contains the same information with respect to the association at issue, only one study will be included. This will be based on the following order: (1) the publication with the largest sample size, (2) the most recent publication or (3) the study that examined KOA using the highest ranked outcome measure. Hierarchy of the definitions is described later at 'Data items, outcomes and hierarchy', and in table 2. If studies of the same study population present different information with respect to the association at issue, both studies will be included. A PRISMA flowchart will be completed to summarise the process. Neither of the review authors will be blinded to the journal titles, the study authors or the institutions. ${ }^{16}$

\section{Data collection process}

Two reviewers (JT and ADI) will independently extract data from the included studies using a customised form, piloted prior to use. Consensus on extracted data will be reached by discussion, and conflictive data extraction will be discussed with a third member of the study team (CJ or HL).

\section{Data items, outcomes and hierarchy}

Data items that will be extracted from included studies are described in table 3. Table 3 shows that in cohort studies, preferably the number of participants developing KOA and not developing KOA, and the number of participants exposed and not exposed to the biomechanical factor will be extracted. In cross-sectional or casecontrol studies, preferably the number of participants with and without KOA, and the number of participants exposed and not exposed to the biomechanical factor will be extracted. If this is not available, mean values for the biomechanical factors or ORs will be extracted, respectively. The same data extraction and analyses will be performed for biomechanical factors and other risk factors. Therefore, in the following paragraphs only biomechanical factors will be mentioned. The hierarchy for definitions of KOA is based on comprehensiveness of the definition and the use in clinical practise (see table 2, ranking: A-J). Symptomatic KOA is based on the 
Table 3 Data extraction

\begin{tabular}{|c|c|}
\hline First au & Age (mean $\pm S D)$ \\
\hline Year of publication & $\mathrm{BMI}($ mean $\pm \mathrm{SD})$ \\
\hline & Definition of KOA used \\
\hline Duration of follow-up* & Definition of BF used \\
\hline Number of participants & Definition of RF used $\dagger$ \\
\hline & $\begin{array}{l}\text { Number of participants } \\
\text { developing } \mathrm{KOA}^{*} \text { or with } \\
\mathrm{KOA} \ddagger\end{array}$ \\
\hline Cohort studies & $\begin{array}{l}\text { Case-control or } \\
\text { cross-sectional studies }\end{array}$ \\
\hline $\begin{array}{l}\text { Number of participants } \\
\text { developing KOA-exposed } \\
\text { to BF/RF }\end{array}$ & $\begin{array}{l}\text { Number of participants with } \\
\text { KOA-exposed to BF/RF }\end{array}$ \\
\hline $\begin{array}{l}\text { Number of participants not } \\
\text { developing KOA-exposed } \\
\text { to BF/RF }\end{array}$ & $\begin{array}{l}\text { Number of controls- } \\
\text { exposed to BF/RF }\end{array}$ \\
\hline Number of participants & Number of participants with \\
\hline developing $\mathrm{KOA}-n o t$ & $\mathrm{KOA}-$ not exposed to BF/ \\
\hline & \\
\hline $\begin{array}{l}\text { Number of participants not } \\
\text { developing KOA-not } \\
\text { exposed to BF/RF }\end{array}$ & $\begin{array}{l}\text { Number of controls-not } \\
\text { exposed to BF/RF }\end{array}$ \\
\hline or & \\
\hline $\begin{array}{l}\text { Mean } \pm \text { SD of biomechanical } \\
\text { factor within participants } \\
\text { developing KOA }\end{array}$ & $\begin{array}{l}\text { Mean } \pm S D \text { of biomechanical } \\
\text { factor within participants } \\
\text { with KOA }\end{array}$ \\
\hline $\begin{array}{l}\text { Mean } \pm S D \text { of biomechanical } \\
\text { factor within participants not } \\
\text { developing KOA }\end{array}$ & $\begin{array}{l}\text { Mean } \pm S D \text { of biomechanical } \\
\text { factor within controls }\end{array}$ \\
\hline or & \\
\hline $\begin{array}{l}\text { ORs for the association } \\
\text { between BF/RF and the } \\
\text { development of KOA }\end{array}$ & $\begin{array}{l}\text { ORs for the association } \\
\text { between BF/RF and KOA }\end{array}$ \\
\hline \multicolumn{2}{|c|}{$\begin{array}{l}\text { Preferably the numbers of participants are extracted. If these are } \\
\text { not available, mean values for the biomechanical or risk factor, or } \\
\text { ORs are extracted, respectively. } \\
\text { ^For cohort studies. } \\
\text { †lf eligible. } \\
\text { †For case-control and cross-sectional studies. } \\
\text { BF, biomechanical factor; KOA, knee osteoarthritis; RF, risk factor }\end{array}$} \\
\hline
\end{tabular}

American College of Rheumatology criteria. $^{17}$ Radiographic KOA is based on radiological atlases (eg, the Kellgren and Lawrence (K/L) Classification). ${ }^{18} 19$ In addition, physicians or authors can state that participants have KOA. The hierarchy will be used for study selection if more than one study examines the same population, and for data extraction in studies using two or more outcome measures of KOA. For the latter, only data regarding the highest ranked outcome measure will be extracted.

\section{Risk of bias in individual studies}

Risk of bias for each included study will be scored independently by two reviewers (JT and ADI). Consensus on conflicting scores will be reached by discussion. The Quality In Prognostic Studies tool will be used. ${ }^{20}$ Six areas of potential study biases will be assessed: study participation, study attrition, prognostic factor measurement, study confounding, outcome measurement and statistical analysis and reporting. Risk of bias for study participation is the likelihood that the relationship between the prognostic factor and the outcome is different for participants and eligible non-participants. Risk of bias for study attrition is the likelihood that the relationship between the prognostic factor and the outcome is different for completing and non-completing participants. Risk of bias for prognostic factor measurement is related to differential measurement of the prognostic factor related to the level of outcome. Risk of bias for study confounding is the effect of the prognostic factor being distorted by another factor that is related to the prognostic factor and outcome. Risk of bias for outcome measurement is related to differential measurement of outcome related to the baseline level of the prognostic factor. Risk of bias for statistical analysis and reporting is the risk of bias whether the statistical analysis is dependent on KOA status and the exposure to the biomechanical factor or not, and whether the presentation of results differs between KOA status and exposure. Study attrition is not applicable for cross-sectional and casecontrol studies, thus will only be rated in cohort studies. Studies will be classified as being of high quality if all study biases are assessed to have a low or moderate risk of bias. Studies with a high risk of bias for at least one study bias will be defined as low-quality studies. A summary statement regarding the quality of the included studies will be reported in the results section.

\section{Assessment of publication bias}

It is assumed that biomechanical projects indicating no association between a biomechanical factor and (the development of) KOA are likely to not be published. Therefore, funnel plots will be used to show the OR on the $\mathrm{x}$-axis against the sample size on the $\mathrm{y}$-axis for each biomechanical factor.

\section{Data synthesis}

Data will be grouped by the category abnormal loading and the main biomechanical impairments. Subsequently, data will be grouped per subdivision of biomechanical impairments as shown in table 1, and per study design (ie, cohort studies vs a combination of cross-sectional and case-control studies). Biomechanical factors studied in more than one study per study design will be subjected to meta-analyses. Meta-analyses will be applied on the OR of developing KOA in participants who are exposed to the biomechanical factor of interest (cohort studies), or the OR of the biomechanical factor being present in participants with KOA compared to the control group (cross-sectional or case-control studies). Random effects model (Mantel Haenszel method) will be used, as large clinical heterogeneity is expected due to the variation in the definition of KOA and biomechanical factors. Metaregression analyses using study-level 
risk factors as covariates in a multivariate regression analysis can be used to explore the impact of different biomechanical factors. ${ }^{21}$ The impact of the biomechanical factors will be assessed by fitting multiple restricted maximum likelihood-based metaregression models. ${ }^{22} 23$ A priori, we defined a relevant covariate (biomechanical or other risk factor) as one that would decrease the between-study variance (estimated as tau-squared $\left(\tau^{2}\right)$, as a consequence of the inclusion in the meta-regression analysis. ${ }^{24}$ Meta-regression analysis will also be used to assess the impact of other risk factors, combinations of other risk factors, including interactions between risk factors, on the association of biomechanical factors with (the development of) KOA. A forest plot will be made for each biomechanical factor, and for combinations of biomechanical factors and other risk factors.

Heterogeneity between studies combined in one meta-analysis will be examined with standard Q-tests, and will be calculated as the $\mathrm{I}^{2}$ statistics, measuring the proportion of inconsistency in the summary effect measure due to between-study heterogeneity. ${ }^{25} 26$

If any substantial heterogeneity will be identified through analysis of $\chi^{2}$ and $\mathrm{I}^{2}$ statistics, subgroup and sensitivity analyses will be performed. These will only be performed if at least three studies are included in the meta-analysis. Subgroup analyses will be used to explore possible sources of heterogeneity. Subgroups will be based on the outcome definition used, that is, clinical, radiological, MRI and surgical criteria. Subgroups will also be identified based on the ways to measure the biomechanical factor and the duration of followup (cohort studies). Other subgroups will be tibiofemoral osteoarthritis and general KOA, and medial and lateral tibiofemoral osteoarthritis. Sensitivity analyses will be carried out based on risk of bias. If sensitivity analyses appear to influence the findings of the review, this will be reported and discussed in the 'Discussion' section.

\section{Confidence in cumulative estimate}

The quality of evidence will be evaluated for the association of the category abnormal loading, and each subdivision of a biomechanical impairment with the (development of) KOA, because this relates to different assessment strategies for clinicians and healthcare providers. The Grading of Recommendation, Assessment, Development and Evaluation framework adapted for prognostic studies will be used. ${ }^{27}$ Factors that may decrease the quality level of evidence are phase of investigation, study limitations, inconsistency, indirectness, imprecision and publication bias. Factors that may increase the quality level of evidence are moderate or large effect size and exposure-response gradient. It is proposed to base the starting point for the quality level of evidence on phase of investigation. This is not applicable for cross-sectional and case-control studies. Therefore, the starting point for these studies will be low. Quality level of evidence will be determined as high, moderate, low or very low, and will be reported in the summary of findings table. Quality of evidence will also be assessed for combinations of biomechanical factors and risk factors that will be examined in meta-analyses.

An algorithm will be developed that can be used by healthcare providers to identify the biomechanical risk factors which are present in persons at high risk of developing KOA. ${ }^{28}$ Biomechanical factors will be divided into categories base on study type (longitudinal and crosssectional/case-control studies) and base of evidence. Evidence will be based on a significant meta-analysis, a longitudinal or cross-sectional/case-control study with a significant finding, an insignificant meta-analysis, or on a longitudinal or cross-sectional/case-control study with insignificant findings. This results in different categories within the algorithm; must consider (significant meta-analysis), maybe consider (study with significant finding), do not consider (insignificant meta-analysis), and not currently clinically relevant (study with insignificant findings).

\section{ETHICS AND DISSEMINATION}

This article describes the framework for a systematic review and meta-analysis into the association of biomechanical factors with the development of KOA. To the best of our knowledge, performing this will result in the first review that gives an overview of the existing evidence of biomechanical factors that are associated with the development of KOA. This systematic review and meta-analysis protocol does not require ethical approval. The results of this systematic review and meta-analysis will be disseminated via publications in peer-reviewed journals, presentations at (inter)national conferences and patient information.

\section{Author affiliations}

${ }^{1}$ Research Unit for Musculoskeletal Function and Physiotherapy, Department of Sports Science and Clinical Biomechanics, Faculty of Health Sciences, University of Southern Denmark, Odense, Denmark

${ }^{2}$ Institute of Applied Health Research, Glasgow Caledonian University, Glasgow, Scotland

${ }^{3}$ Department of Rehabilitation, Copenhagen University Hospital, Herlev and Gentofte, Denmark

${ }^{4}$ Department of Rehabilitation Medicine and Department of Psychiatry, EMGO Institute for Health and Care Research, VU University Medical Center, Amsterdam, The Netherlands

${ }^{5}$ Centre for Evidence-Based Practice, Bergen University College, Bergen, Norway

\section{Twitter Follow Joyce van Tunen at @JvTunen}

Contributors JT, ADI, CJ, JD, MS and HL drafted the protocol. JT, ADI, CJ and $\mathrm{HL}$ contributed to the development of the selection criteria, the risk of bias assessment strategy and data extraction criteria. JT and $\mathrm{HL}$ developed the search strategy. JT, CJ and HL provided statistical expertise. All authors read, provided feedback and approved the final protocol. JT is the guarantor of this article.

Funding The research leading to these results has received funding from the European Union Seventh Framework Programme (FP7-PEOPLE-2013-ITN; KNEEMO) under grant agreement $n$ 607510. This work was also supported by the Open Access Fund from the University of Southern Denmark.

Competing interests None declared.

Provenance and peer review Not commissioned; externally peer reviewed. 
Open Access This is an Open Access article distributed in accordance with the Creative Commons Attribution Non Commercial (CC BY-NC 4.0) license, which permits others to distribute, remix, adapt, build upon this work noncommercially, and license their derivative works on different terms, provided the original work is properly cited and the use is non-commercial. See: http:// creativecommons.org/licenses/by-nc/4.0/

\section{REFERENCES}

1. Brandt KD, Radin EL, Dieppe PA, et al. Yet more evidence that osteoarthritis is not a cartilage disease. Ann Rheum Dis 2006;65:1261-4.

2. Silverwood V, Blagojevic-Bucknall $M$, Jinks $C$, et al. Current evidence on risk factors for knee osteoarthritis in older adults: a systematic review and meta-analysis. Osteoarthr Cartil 2015;23:507-15.

3. Zhang W. Risk factors of knee osteoarthritis-excellent evidence but little has been done. Osteoarthr Cartil 2010;18:1-2.

4. Knoop J, Steultjens MPM, van der Leeden M, et al. Proprioception in knee osteoarthritis: a narrative review. Osteoarthr Cartil 2011;19:381-8.

5. Oiestad BE, Juhl CB, Eitzen I, et al. Knee extensor muscle weakness is a risk factor for development of knee osteoarthritis. A systematic review and meta-analysis. Osteoarthr Cartil 2015;23:171-7.

6. Lewek MD, Rudolph KS, Snyder-Mackler L. Control of frontal plane knee laxity during gait in patients with medial compartment knee osteoarthritis. Osteoarthr Cartil 2004;12:745-51.

7. Sharma L, Lou C, Felson DT, et al. Laxity in healthy and osteoarthritic knees. Arthritis Rheum 1999;42:861-70.

8. Guilak F. Biomechanical factors in osteoarthritis. Best Pract Res Clin Rheumatol 2011;25:815-23.

9. Kerkhof $\mathrm{HJ}$, Bierma-Zeinstra SM, Arden NK, et al. Prediction model for knee osteoarthritis incidence, including clinical, genetic and biochemical risk factors. Ann Rheum Dis 2014;73:2116-21.

10. Moyer RF, Ratneswaran A, Beier F, et al. Osteoarthritis year in review 2014: mechanics-basic and clinical studies in osteoarthritis. Osteoarthr Cartil 2014;22:1989-2002.

11. Tanamas S, Hanna FS, Cicuttini FM, et al. Does knee malalignment increase the risk of development and progression of knee osteoarthritis? A systematic review. Arthritis Rheum 2009;61:459-67.
12. Moher D, Booth A, Stewart L. How to reduce unnecessary duplication: use PROSPERO. BJOG 2014;121:784-6.

13. Shamseer L, Moher D, Clarke M, et al. Preferred reporting items for systematic review and meta-analysis protocols (PRISMA-P) 2015: elaboration and explanation. BMJ 2015;349:g7647.

14. Moher D, Liberati A, Tetzlaff J, et al. Preferred reporting items for systematic reviews and meta-analysis: the PRISMA statement. PLoS Med 2009;6:e1000097.

15. Rathbone J, Carter M, Hoffmann T, et al. Better duplicate detection for systematic reviewers: evaluation of Systematic Review Assistant-Deduplication Module. Syst Rev 2015;4:6

16. Berlin JA. Does blinding of readers affect the results of meta-analyses? Lancet 1997;350:185-6.

17. Altman R, Asch E, Bloch D, et al. Development of criteria for the classification and reporting of osteoarthritis: classification of osteoarthritis of the knee. Arthritis Rheum 1986;29:1039-49.

18. Altman RD, Gold GE. Atlas of individual radiographic features in osteoarthritis, revised. Osteoarthr Cartil 2007;15(Suppl A):A1-56.

19. Culvenor AG, Engen CN, Oiestad BE, et al. Defining the presence of radiographic knee osteoarthritis: a comparison between the Kellgren and Lawrence system and OARSI atlas criteria. Knee Surg Sport Traumatol Arthrosc 2015;23:3532-9.

20. Hayden JA, van der Windt DA, Cartwright JL, et al. Assessing bias in studies of prognostic factors. Ann Intern Med 2013;158:280-6.

21. Thompson SG, Higgins JPT. Treating individuals 4: can meta-analysis help target interventions at individuals most likely to benefit? Lancet 2005;365:341-6.

22. Thompson SG, Sharp SJ. Explaining heterogeneity in meta-analysis: a comparison of methods. Stat Med 1999;18:2693-708.

23. Thompson SG, Higgins JP. How should meta-regression analyses be undertaken and interpreted? Stat Med 2002;21:1559-73.

24. van Houwelingen $\mathrm{HC}$, Arends LR, Stijnen T. Advanced methods in meta-analysis: multivariate approach and meta-regression. Stat Med 2002;21:589-624.

25. Higgins JP, Thompson SG. Quantifying heterogeneity in a meta-analysis. Stat Med 2002;21:1539-58.

26. Higgins JP, Thompson SG, Deeks JJ, et al. Measuring inconsistency in meta-analyses. BMJ 2003;327:557-60.

27. Huguet A, Hayden JA, Stinson J, et al. Judging the quality of evidence in reviews of prognostic factor research: adapting the GRADE framework. Syst Rev 2013;2:71

28. Aderem J, Louw QA. Biomechanical risk factors associated with iliotibial band syndrome in runners: a systematic review. BMC Musculoskelet Disord 2015;16:356. 